\title{
COCCIDIOSE HEPÁTICA EM Aspistor quadriscutis, Valenciennes, 1840 (TELEOSTEI: ARIIDAE) CAPTURADOS NO ESTUÁRIO DO NORDESTE PARAENSE, AMAZÔNIA, BRASIL.
}

\author{
(Hepatic coccidiosis in Aspistor quadriscutis, valenciennes, 1840 (teleostei: ariidae) \\ captured in the estuary of the northeast paraense, amazonia, brazil.))
}

\author{
Ana Beatriz Mendes Amaral Ramos ${ }^{1 *}$, Patrícia de Fátima Saco dos Santos ${ }^{1}$, Lenize Carolina Alves \\ Hemeterio ${ }^{1}$, Weverton John Pinheiro dos Santos ${ }^{1}$, Rebeca Silva de Araújo², Edilson Rodrigues Matos ${ }^{1}$ \\ 1Universidade Federal Rural da Amazônia - UFRA, Brazil. *Corresponding author: abmaramos@gmail.com \\ 2 Universidade Federal do Acre - UFAC
}

RESUMO: O consumo de pescado contaminado pode causar sérios danos à saúde do consumidor. O gênero Calyptospora abarca uma variedade de formas parasitárias que podem incidir em peixes, aves e moluscos bivalves. Durante esse estudo foram coletados 38 espécimes de Aspistor quadriscutis (bagre ou bagrebranco) no estuário de São Caetano de Odivelas, na microrregião do Salgado. Os peixes foram capturados, transportados ao Laboratório de Pesquisa Carlos Azevedo - LPCA/UFRA, e necropsiados para análise da região hepática. Os lobos hepáticos apresentavam manchas acinzentadas. Retirou-se fragmentos da região para observar a morfologia tecidual e verificar a presença de parasitos. Foram encontrados vacúolos parasitóforos, contendo quatro esporocistos e em cada, dois esporozoítos, como características dos coccídios, gênero Calyptospora. Em seguida, o fígado foi colhido e fixado em solução de Davidson, desidratado em série alcoólica crescente, diafanizado em xilol, impregnado em parafina líquida e incluídos para o posterior corte histológico. Com cortes de $5 \mu$ os tecidos foram corados em Ziehl-Neelsen e Hematoxilina\&Eosina, sendo montados em lâmina/lamínula com entellan, para confirmação dos achados patológicos.

Palavras-chave: bagre, Calypstospora sp., região hepática, histologia.

\begin{abstract}
Consumption of contaminated fish can cause serious harm to consumer health. The genus Calyptospora comprises a variety of pathogenic and parasitic forms that can affect fish, birds and bivalve molluscs. A total of 38 specimens of Aspistor quadriscutis (Bagre or Bagre-Branco) were collected in the estuary of the municipality of São Caetano de Odivelas, in the Salgado micro-region. The fish were captured and taken to the Carlos Azevedo Research Laboratory LPCA / UFRA, and then necropsied for further analysis of the hepatic region. The hepatic lobes had grayish spots. Parasites with rounded morphology were observed in the hepatic tissue and, internally, with four ellipsoid structures, called sporocysts. Inside each sporocysts there are two sporozoites, which characterizes coccidiosis, genus Calyptospora. After the liver was harvested, fixed in Davidson, dehydrated in increasing alcoholic series, diaphanized in xylol, impregnated with liquid paraffin, included for posterior histological cut. After cutting, the tissues were stained in the Ziehl-Neelsen and Hematoxylin \& Eosin stains, and mounted on a slide with entellan, to confirm the pathological findings.
\end{abstract}

Keywords: bagre, Calypstospora sp., hepatic region, histology. 


\section{INTRODUÇÃO}

São Caetano de Odivelas faz parte de um complexo de estuários localizado no nordeste paraense, que recebe influência do Rio Mojuim e deságua no oceano Atlântico (BARROS, 2011). A ictiofauna estuarina é extremamente diversificada, porém 0 conhecimento científico desta na região amazônica ainda é limitado (BARROS, 2011). São conhecidas apenas 303 espécies, pertencentes a 23 ordens e 86 famílias, de acordo com estudos realizados nos estados no Pará e Amapá (Camargo et al., 2001).

A espécie Aspistor quadriscutis (Bagre-Branco ou Bagre) faz parte desta ictiofauna, pertencente à ordem Siluriforme, família Ariidae, representando $80 \%$ da biomassa total de pescado capturado (Soares et al., 2016). O filo Apicomplexa Levine, 1970 inclui vários patógenos de importância médica e médico-veterinária. É caracterizado por apresentar complexo apical, composto de organelas secretoras especializadas (Souza et al., 2010). Pertence ao reino Protozoa e infecta vários grupos, parasitando também peixes, moluscos e bivalves (Matos et al., 2004). A família Calyptosporidae foi descrita em peixes (Overstreet et al., 1984) e engloba o gênero Calyptospora, que tem, em sua maioria, a região hepática de peixes como sítio de infecção (Fournie et al., 1985). Atualmente são conhecidas três espécies do gênero Calyptospora que infectam peixes: Calyptospora serrasalmi (Cheung et al., 1986), infectando Serrasalmus nigers; Calyptospora tucunarensis, que tem como hospedeiro a espécie Cichla ocellaris (Békési et al., 1991); e Calyptospora spinosa, encontrado em Crenicicha lepidota, espécie de peixe amazônico (Azevedo et al., 1993).
Morfologicamente a família Calyptosporidae é caracterizada por apresentar vacúolo parasitóforo, com quatro esporocistos, e dentro destes, dois esporozoítos. Os esporocistos podem variar de morfologia arredondada a elipsoide (Azevedo et al., 1995). Quando há um alto grau de infecção, cerca de $85 \%$ do fígado do hospedeiro pode ser substituído por agregações de oocistos, deixando o tecido com lesões pretas e com uma pigmentação branca pálida (Lom et al., 1992).

O presente estudo busca identificar morfologicamente através da histologia, parasitos do gênero Calypstopora encontrados na região hepática de Aspistor quadriscutis, no estuário amazônico.

\section{MATERIAL E MÉTODOS}

Foram coletados 38 espécimes de $A$. quadriscutis (Bagre ou BagreBranco) no estuário do município de São Caetano de Odivelas, na microrregião do Salgado $\left(00^{\circ} 45^{\prime} S\right.$ ;4801' O) (figura 1). Os peixes foram capturados com linha de pesca. Após a captura, os animais vivos, foram acondicionados em sacos com aeração artificial e transportados até 0 Laboratório de Pesquisa Carlos Azevedo - LPCA/ UFRA, colocados em aquários para posterior necrópsia. Os peixes foram anestesiados utilizando Tricaína Metano sulfonato (MS222 SIGMA) na concentração de $50 \mathrm{mg} / \mathrm{L}$ e/ou mielotomia neural conforme 0 protocolo do CEUA-UFRA 013/2014 e seguindo a licença do SISBIO- ICMBIO no 27119-1. Durante a necrópsia foram retirados fragmentos do fígado do animal para observação na microscopia de luz (microscópio OLYMPUS CX41, utilizando as objetivas de 4X, 10X e 
40X) e processamento histológico. O tecido foi fixado em solução de Davidson (ácido acético, álcool 95\%, formol e água destilada) por 24h. $\mathrm{Na}$ sequência o tecido foi processado, desidratado (série crescente de álcool), diafanizado em xilol, impregnado por parafina líquida, e foi realizada a inclusão (TISSUE EMBEDDING CENTER MICRON EC350). Cortes com $0,5 \mu \mathrm{m}$ de espessura foram feitos e corados em H\&E (Hematoxilina-Eosina) e Ziehl-Neelsen. Os tecidos corados foram fotografados utilizando microscópio óptico ZEISS PRIMO STAR e uma câmara acoplada ZEISS AXIO CAM ERC 5S, com software AXIOVISION LE 4.8.2.

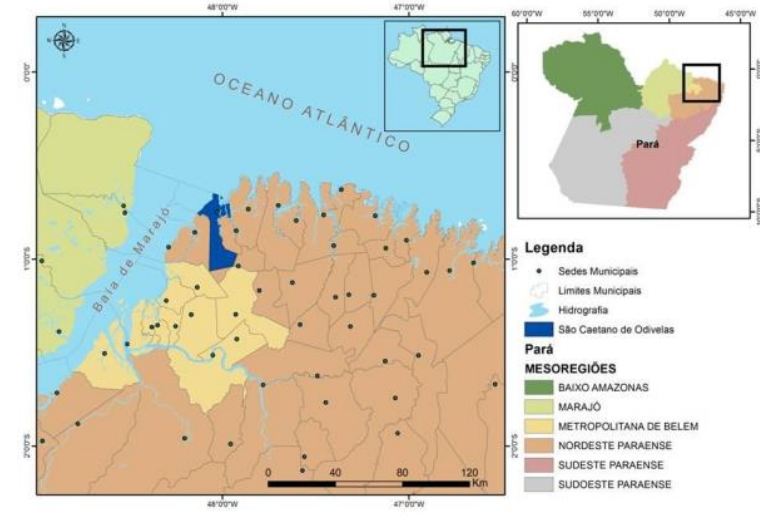

Figura 1: Localização geográfica do município de São Caetano de Odivelas

\section{RESULTADOS E DISCUSSÃO}

Ao observar a região hepática após a necrópsia, o órgão estava com coloração esbranquiçada e manchas acinzentas nos lobos hepáticos, corroborando os resultados obtidos por Cheung et al. (1986) e Velasco et al. (2012). Ao retirar fragmentos do fígado para observação na microscopia de luz (microscópio OLYMPUS CX41), foi confirmada a presença de oocistos, e que as lesões acinzentadas foram causadas pela ação desse parasito. Os parasitos se apresentavam de forma isolada ou agrupados (figura 2), difundidos por todo tecido, como 0 descrito por Velasco et al. (2012), em Cichla temensis.

Estes parasitos têm como característica morfológica um oocisto arredondando, no interior do qual existem quatro esporocistos elipsoides, e dentro de cada um, dois esporozoítos, características do gênero Calyptospora, confirmando os achados de Albuquerque e Brasil Sato (2010), Bonár et al. (2006), Fournie et al. (2000), Silva et al. (2012), Velasco et al. (2012) e Whipps et al. (2012).

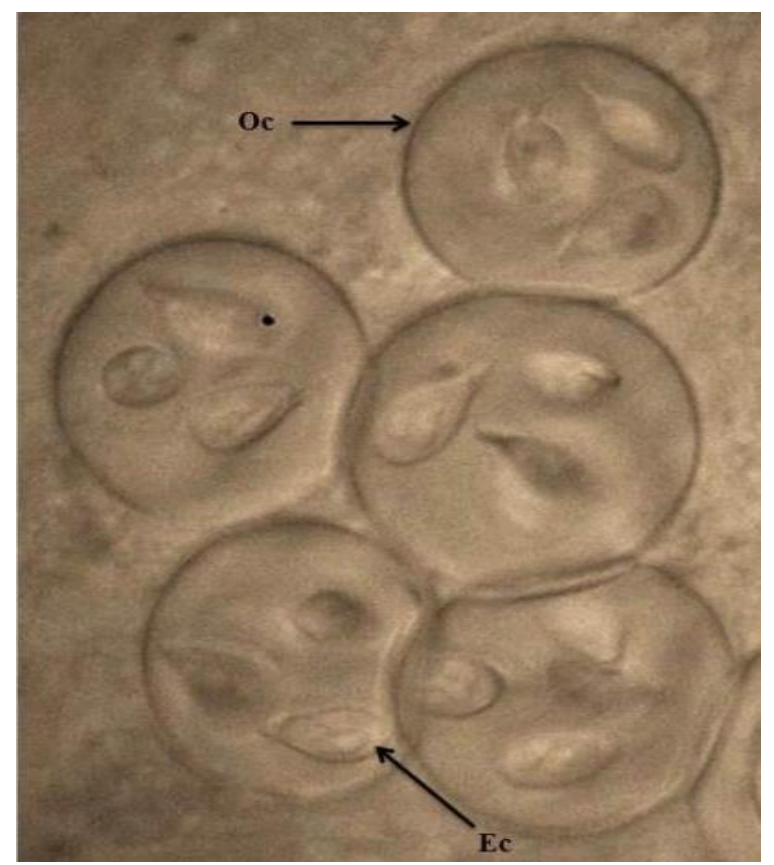

Figura 2: Oocistos a fresco de Calypstopora sp. Oc-oocitos. Ec - esporocistos

A prevalência parasitária foi de $84,21 \%$, com espécimes infectados em todos os períodos de coleta. Esta prevalência difere das achadas por Azevedo et al. (1993), Békési e Molnár (1991), Silva et al. (2012) e Velasco et al. (2012).

Foram evidenciados na análise histológica vários aglomerados no parênquima hepático (figuras 3 e 4), ocorrendo uma degeneração no fígado e a ausência de inflamação significativa do tecido hepático, indo ao encontro do que foi descrito por Békési e Molnár (1991), Solangi e Overstreet (1980) e Videira et al. (2013). 
Apesar da grande carga parasitária encontrada na região hepática, verificou-se a ausência de inflamação no tecido. As características morfológicas apresentam um importante passo para a identificação do parasito, porém fazem-se necessárias aplicações de métodos morfométricos e moleculares (Berto et al., 2014).

Este é o primeiro registro de infecção por Calypstospora sp. em região hepática de $A$. quadriscutis.

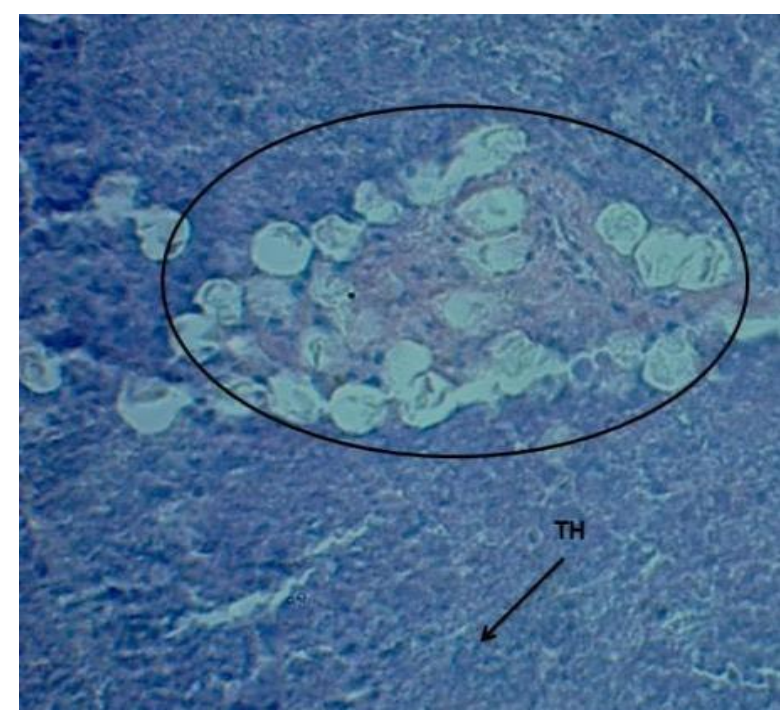

Figura 3: Aglomerado de oocisto na região hepática de $A$. quadriscutis. $(\rightarrow)$ TH - Tecido Hepático, região sem oocistos (em azul). Coloração Ziehl-Neelsen.

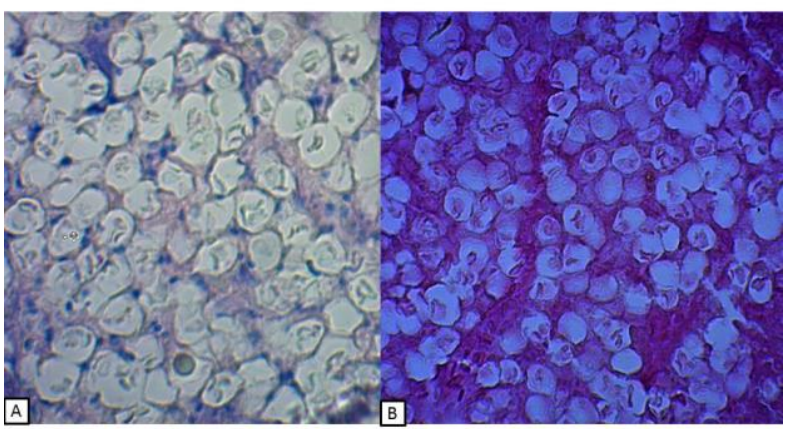

4: A - Observação geral do tecido, utilizando a técnica de coloração Hematoxilina-Eosina (10X). B - Coloração em Ziehl-Neelsen do tecido hepático infestado de Calyptospora $s p$, observando o alto grau de parasitismo (400X).

\section{CONCLUSÃO}

Estudos complementares estão sendo realizados para a confirmação da espécie do parasito encontrado, pois deverá ser nova espécie, demonstrada por análises em biologia molecular.

\section{AGRADECIMENTOS}

Á Universidade Federal Rural da Amazônia (UFRA), à FAPESPA, CAPES, ao CNPq e à Universidade Federal do Pará (UFPA).

\section{REFERÊNCIAS}

ALBUQUERQUE, M.C.; BRASIL-SATO, M.C. First report of Calyptospora sp.(Apicomplexa, Calyptosporidae) in forage characid fish from the Três Marias Reservoir, São Francisco Basin, Brazil. Eur J Protistol, v.2, n.6, p.150152, 2010.

AZEVEDO, C.; MATOS, P.; MATOS, E. Morphological data of Calyptospora spinosa n. sp. (Apicomplexa, Calyptosporidae) parasite of Crenicichla lepidota Heckel, 1840 (Teleostei) from Amazon River. Eur J Protistol, v.2, n.9, p.171-175, 1993.

AZEVEDO, C.; MATOS, P.; MATOS, E. Ultrastructural data on sporogony of the coccidian parasite Calyptospora spinosa from the liver of the Amazonian fish, Crenicichla lepidota Heckel. J Fish Dis, v.6, n.18, p. 475-479, 1995.

BARROS, D.F.; TORRES, M.F.; FRÉDOU, F.L. Ichthyofauna of the estuary of São Caetano de Odivelas and Vigia (Pará, Amazon Estuary). Biota Neotrop, v.2, n.11, 2011.

BÉKÉSI, L.; MOLNÁR, K. Calyptospora tucunarensis n. sp. (Apicomplexa: Sporozoea) from liver of tucunare Cichla ocellaris in Brazil. SystParasito, v.2, n.18, p.127-132, 1991 
BERTO, B.P.; MCINTOSH, D.; LOPES, C.W.G. Studies on coccidian oocysts (Apicomplexa: Eucoccidiorida). Rev. Bras. Parasitol, Vet. v.23, n.1, 2014.

BONAR, C.; POYNTON, S.L.; SCHULMA, F.Y, RIETCHECK, RL.; GARNER, M.M. Hepatic Calyptospora sp. (Apicomplexa) infection in a wildborn, aquarium-held clutch of juvenile arapaima Arapaima gigas (Osteoglossidae). Dis Aquat Organ, v.1-2, n.70, p.81-92, 2006.

CHEUNG, P.J.; NIGRELLI, R.F.; RUGGIERI, G.D. Calyptospora serrasalmi sp. nov. (Coccidia: Calyptosporidae) from liver of the black piranha, Serrasalmus niger Schomburgk. J Aquaricult Aquat Sci, v.3, n.4, p.54-57, 1986.

LOM, J.; DYKOVÁ, I .Protozoan parasites of fishes. Elsevier: New York, 1992.

MARCENIUK, A.P.; FERRARIS JR, C.J. Checklist of the freshwater fishes of South and Central America. Ariidae (Sea catfishes). Porto Alegre: EDIPUCRS, Brasil, p.447-455, 2003.

OVERSTREET, R.M.; HAWKINS, W.E.; FOURNIE, J.W. The coccidian genus Calyptosporan. g. and Family Calyptosporidae n. fam. (Apicomplexa), with members infecting primarily fishes. J Eukaryot Microbiol, v.2, n.31, p.332339, 1984.

SILVA, M.C.; SÃO CLEMENTE, S.C.; PICANÇO JR, J.A; VELASCO, M.; MATOS, E.R. Calyptospora sp. in Brachyplatystoma vaillantii trapped at the Vigia, State of Pará, Brazil. Rev. Bras. Parasitol, Vet., Jaboticabal, v. 21, n. 2, p. 176-178, 2012.
SOLANGI, M.A.; OVERSTREET, T.M. Biology and pathogenesis of the coccidium Eimeria funduli infecting killifishes. J. Parasitol, n.66, p.513-526, 1980.

SOARES, B. E.; SANTOS, A. C. C.; MENDES, F. L. S.; BARTHEM, R. B.; MONTAG, L. F. A. Length-weight relationship of seven marine catfishes (Siluriformes: Ariidae) in the Amazon Coastal Zone (Brazil). J. Appl. Ichthyol, n.32, p.163-164, 2016.

SOUZA, V.; DUARTE, E.S.M.; LEMGRUBER, L.; ATTIAS, M.; VOMMARO, R.C. Structural organization of the tachyzoite of Toxoplasma gondii. Scientia Medica, v.20, n.1, p. 131-143, 2010.

VELASCO, M.; VIDEIRA, M.; MATOS, P.; SÃO CLEMENTE, S.C.; SANCHES, O.; MATOS, E. Morfologia e nova ocorrência de um coccídio hepatopancreático parasita de peixe amazônico. Rev. Cienc. Agrar, v. 55, n. 3, p. 231-235, 2012.

VIDEIRA, M.; VELASCO, M.; TORTELLY, R.; MENEZES, R.C.; SÃO CLEMENTE, S.C, MATOS, E. An anatomopathological study of hepatic coccidiosis (Calyptospora sp.) in the Acará-pixuna, Aequidens plagiozonatus Kullander, 1984 from the Brazilian state of Pará. Arq. Bras. Med. Vet. Zootec, v.65, n.1, p.91-94, 2013.

WHIPPS, C. M.; FOURNIE, J. W.; MORRISON, D. A.; AZEVEDO, C.; MATOS, E.; THEBO, P.; KENT, M. L. Phylogeny of fish-infecting Calyptospora species (Apicomplexa: Eimeriorina). Parasitology Research, v. 111, p. 1331-1342, 2012. 